\title{
Automatic Guidance of a Tractor Using Computer Vision
}

\author{
Pedro Moreno Matías and Jaime Gómez Gil \\ University of Valladolid, Departament of Teory Signal, Comunications and Telematic \\ Engineering, de Teoría de la Señal, Comunicaciones e Ingeniería Telemática, \\ Campus Miguel Delibes, 47011, Valladolid, \\ Fax: 983423667 \\ jgomez@tel.uva.es
}

\begin{abstract}
This paper presents a computer vision guidance system for agricultural vehicles. This system is based on a segmentation algorithm that uses an optimum threshold function in terms of minimum quadratic value over a discriminant based on the Fisher lineal discriminant. This system has achieved not only very interesting results in the sense of segmentation, but it has also guided successfully a vehicle in a real world environment.
\end{abstract}

\section{Introduction}

This paper describes an automatic agricultural computer vision guided system. Precision Agriculture is defined as the application of technical advances to agriculture; this field has grown rapidly in recent years because it offers a cheaper and more effective way of working the land. When using precision techniques it is more appropriate to use a local rather than a global reference due to benefits as position independence, a priori terrain knowledge independence and tolerance independence [1].

We have chosen a concrete local reference technique that has been rapidly developed for agricultural purpose: Computer Vision. The first tentative trials were direct applications of the traditional computer vision techniques like FFT convolution filters, basic border detection or region division techniques [1], [2]. The Hayashi and Fujii system based on border detection and Hough transform [3] and the system based on texture and intensity from the group of Chateau [4] could be some examples. Lately, some investigators like Reid and Searcy [5], Gerrish [6] and Klassen [7] have developed quite effective systems which were never actually tested guiding a vehicle. In recent years, some systems have achieved great results when guiding a vehicle in a very specific task, like the Ollis and Stentz algorithm [8] for harvesting or the Bulanon, Kataoka, Ota and Hiroma [9] guidance system to collect apples. However, there is still no effective solution to guide an agricultural vehicle with independence of the task.

\section{Objectives}

As mentioned above, the main goal for this project is to develop the most generalpurpose solution possible. Thus its success must be measured in terms of the number 
of agricultural tasks that can be guided by the system. The concrete effectiveness for each concrete task is less important.

The decision of whether a task can be reasonably done or not with the system will depend on the relative distance between the actual division line between areas and the approximation of the algorithm for the segmentation algorithm, and the real distance between rows in the guidance algorithm.

\section{Development}

As described, the system is based on a software application that decides the direction to be taken by the tractor and a hardware interface that communicates with the hardware guidance system. The most complex and interesting part of the system is the software application that implements the computer vision logic. The software is based on the algorithm of Ollis and Stentz [8], where the Fisher lineal discriminant [10], a mathematic tool, was introduced. This software is completed with a simple guidance algorithm.

\subsection{Image Segmentation: The Ollis and Stentz Algorithm}

The algorithm of Ollis and Stentz is row by row processing algorithm that is based on the following assumptions: the main idea is that the image is composed by a two well differentiated areas that have a frontier between the areas inside the image for every row. Along with this, the discriminant, the function that translates the $3 \mathrm{D}$ matrix that composes the image into a 1D matrix which is used to separate the two areas, is a bimodal function. This means that for any of the two differentiated areas in the image, there are presented values of the returning values of the discriminant around a medium value, $\mathrm{m} 1$ and $\mathrm{m} 2$, respectively.

Based on these suppositions, the algorithm is defined as a row by row processing algorithm because each border point can be calculated from the value of the discriminant of the points of the row. In Fig. 1, it is represented an example representation of the discriminant function against the position in the row.

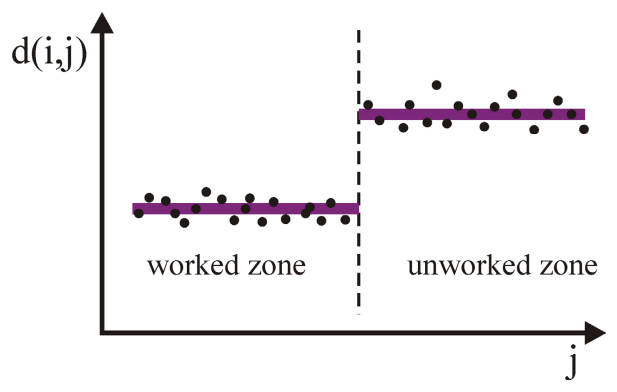

Fig. 1. Threshold example for a discriminant 
With a bimodal discriminant function, the success of the algorithm depends on the correct election of the threshold function in term of MSE (Mean square error). Mathematically, the discriminant can be expressed as the following (1).

$$
d(i, j)=\alpha \cdot r(i, j)+\beta \cdot g(i, j)+\gamma \cdot b(i, j)
$$

\subsection{Discriminant Election: The Fisher Lineal Discriminant}

Although the Ollis and Stentz algorithm was originally defined for a static discriminant, it can be variable in time, so it can be autooptimized as the Fisher lineal discriminant, which is the discriminant chosen for the system described in the paper.

Operatively, the Fisher discriminant is an adaptative stocastic tool obtained from the means and the variances of each area of the image. Concretely, if we analyze the discriminant as the geometric projection of the three colour spaces (one for each component RGB) over an hypothetic line in this 3D space, the Fisher lineal discriminant can be analyzed as the projection of the colour space over the line defined by the vector result of the difference of the two means of each zone. Projecting over this line, it minimizes the overlapping as shown in Fig. 2.

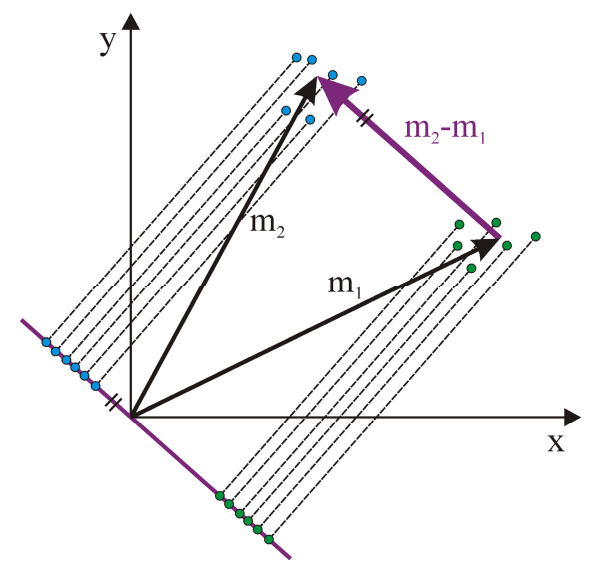

Fig. 2. Graphic example of a Fisher linear discriminant for a 2D colour space

\subsection{Guiding Algorithm}

We have chosen the simplest solution for the guiding algorithm: it consists on an average of the border points detected by the algorithm (which represents implicitly a low-pass filter that eliminates some noise). Although simple, this solution presents good results when the camera is positioned just over the border line and focusing the furthest possible.

For the hardware interface and initial calibration, a lineal approximation between the minimum, centred and maximum positions of the tractor direction and a parallel 
port communication were chosen. This lineal approximation revealed to be more efficient and simpler than the Ackermann algorithm.

\section{Results}

\subsection{Tilling}

In general, tilling is done in times when there is little vegetation, so this limited growth did not affect the results of the experiment; in addition, the conditions of illumination at this time of year were favourable for our trials. Consequently, the obtained segmentation line had a very small deviation from the real line. Nevertheless, the got results were worse when focusing on a point very near the vehicle because the granularity of the image is bigger and the algorithm can be fooled by clumps of earth and small shadows. Fig. 3 shows some examples.
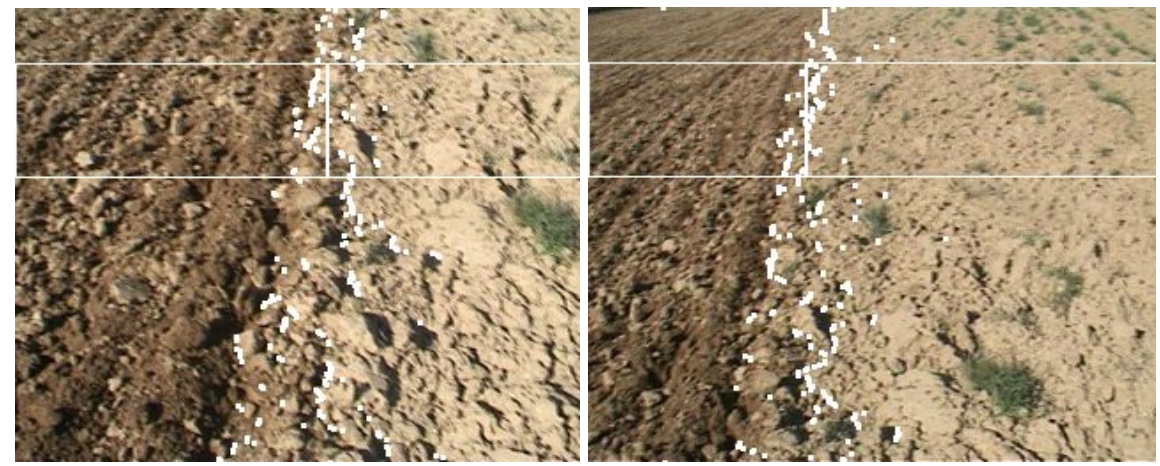

Fig. 3. Tilling examples

\subsection{Harvesting}

We expected harvesting results to be less successful, because of the similarity of the two parts of the image in terms of colour and texture. As the height of the harvested
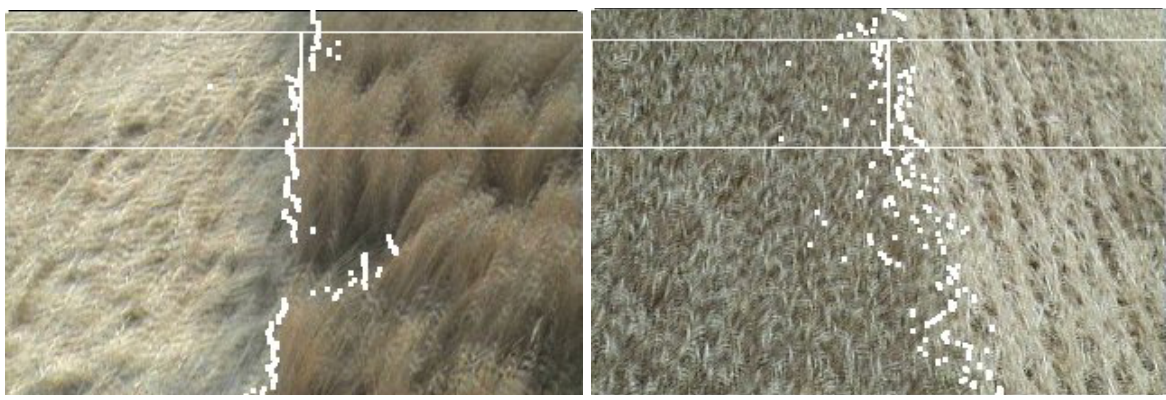

Fig. 4. Harvesting examples 
and uncut plants are so different, the results could be affected by the shadows and light. However, the actual results were quite respectable as shown in Fig. 4.

\subsection{Ploughing and Direct Sowing}

Most of our experiments involved ploughing and direct sowing; therefore, we have a great deal of results. On the one hand, we achieved interesting results even in presumably uninteresting conditions, such as low illumination or low contrast between zones, as shown in Fig. 5.
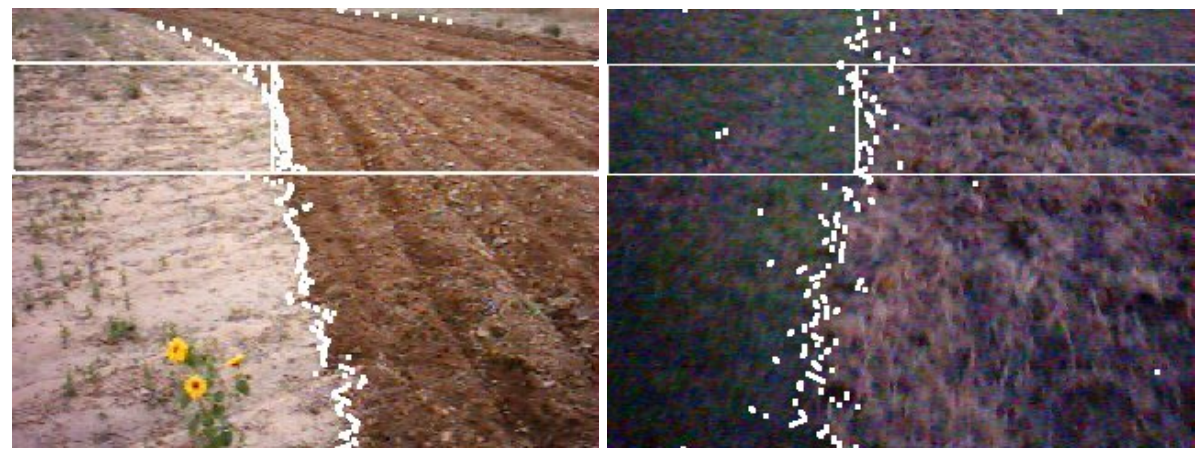

Fig. 5. Correct sowing examples

On the other hand, poor results were also obtained due to shadows and irregular vegetation, where the system completely failed, as can be seen in Fig. 6.
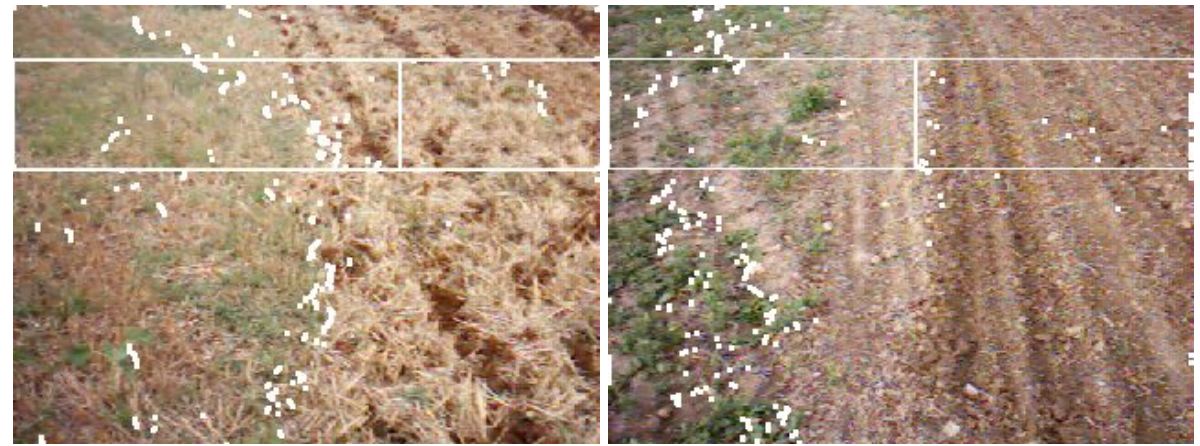

Fig. 6. Incorrect sowing examples

\section{Autoguidance System}

To get an autonomous guidance, it has been installed in a tractor a laptop where it has been connected a webcam (see Fig. 7). 


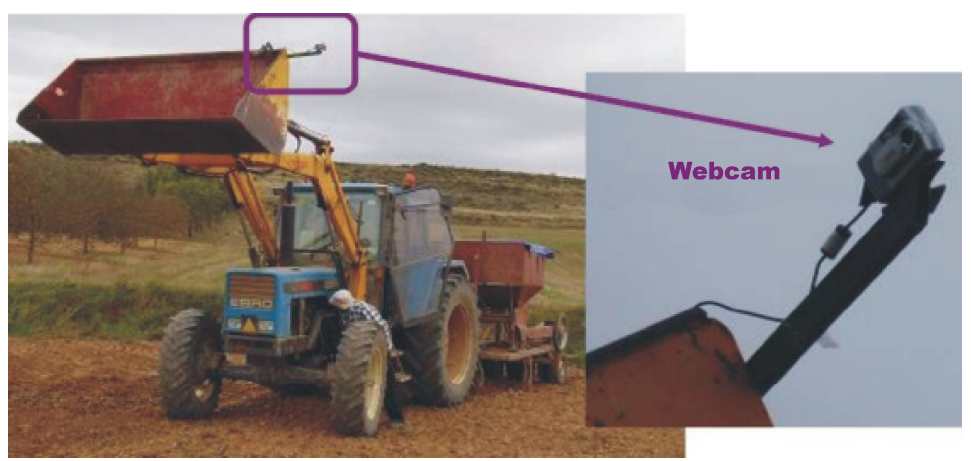

Fig. 7. Installation of the webcam in the tractor

Adaptations in the tractor have had to be done to control the tractor by the notebook which processes the video. It has been installed a controller box which receives data from the notebook about the tractor direction and moves a DC motor which works thedirection. It has also been installed a strap which joins the motor with the steering wheel, a clutch that tightens a strap to pass from manual to autonomous driving and a potentiometer in the front wheel to know in every moment the steering state (see Fig. 8).
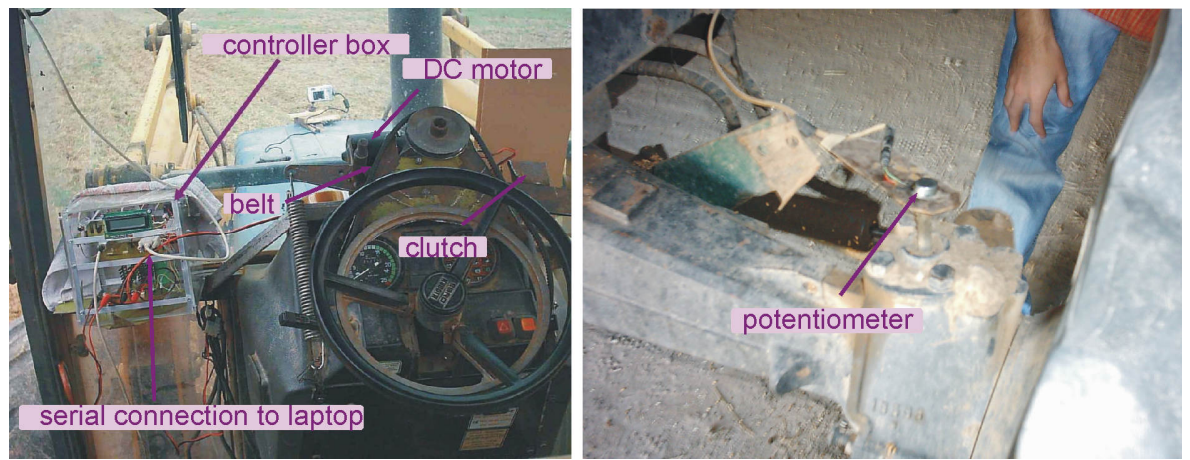

Fig. 8. Left: Adaptations for the movement of the engine. Right: Potentiometer in the front wheel.

Subsequently, tests of autonomous guidance by means of artificial vision with the system presented in this paper have been done. The control law represented in Fig. 9 left has been applied. When the vision system detected the treated area and the nontreated area, the guidance was made correctly. This happened when the tractor raised moist soil with a darker colour. Fig. 9 right presents a plot where the tractor guidance has been made without problems by means of artificial vision. 


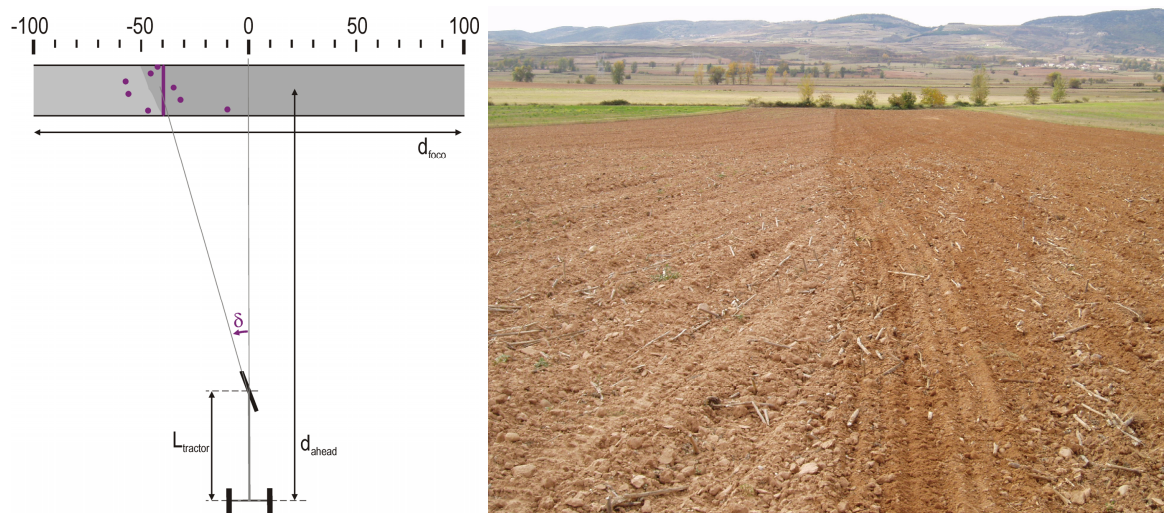

Fig. 9. Left: Graphic representation of the control law. Right: The dark area corresponds with the cultivated area by means of artificial vision. The light area is the non-cultivated area.

\section{Conclusions}

In summary, very positive results have been achieved for every task tested using the system under uniform conditions of illumination and terrain.

However, conditions of non-uniform illumination raise the rate of errors; therefore, the system gets confused by the irregularities shadows of the terrain or external factors.

It can also be observed that the effect of irregular vegetation make the system inefficient when the difference of texture between the processed and non-proccessed areas is not significant, as in tilling. This effect can be reduced with a correct location and focusing of the webcam.

An important result is the good behaviour of the guidance algorithm although it is quite simple. The system has been tested with straight rows but we are sure that with small modifications it would work correctly in curved trajectories.

\section{References}

1. Pajares, G., Cruz, D.1.: Visión por computador: Imágenes digitales y aplicaciones. Ed. RaMa (2001)

2. Jahns, G.: Automatic Guidance in Agriculture: A Review. ASAE paper NCR, St. Joseph. MI, 83-404 (1983)

3. Hayashi, M., Fujii, Y.: Automatic Lawn Mower Guidance Using a Vision System. In: Proceedings of the USA-Japan Symposium on Flexible Automation, New York (1988)

4. Chateau, T.: An Original Correlation and Data Fusion Based Approach to Detect a Reap Limit into a Gray Level Image. In: Proceedings of the 1997 International Conference on Intelligent Robots and Systems, pp. 1258-1263 (1997)

5. Reid, J.F, Searcy, S.W.: Detecting Crop Rows Using the Hough Transform. Presented at the 1986 summer meeting of the ASAE, San Luis Obispo (1986)

6. Gerrish, J.: Path-finding by Image Processing in Agricultural Field Operations. Transactions of the Society of Automotive Engineers (1987). 
7. Klassen: Agricultural Vehicle Guidance Sensor. Paper 93-1008 Presented at the 1993 International Summer Meeting of the ASAE, Spokane, WA (June 1993)

8. Ollis, M., Stentz, A.: First Results in Crop Line Tracking. In: Proceedings of IEEE Conference on Robotics and Automation (ICRA 1996), pp. 951-956. IEEE Computer Society Press, Los Alamitos (1996)

9. Bulanon, D.M., Kataoka, T., Ota, Y., Hiroma, T.: A Segmentation Algorithm for the Automatic Recognition of Fuji Apples at Harvest. Biosystems Engineering 83, 405-412 (2002)

10. Duda, R., Hart, P.: Pattern Classification and Scene Analysis, pp. 114-118. Wiley \& Sons, Chichester (1973) 